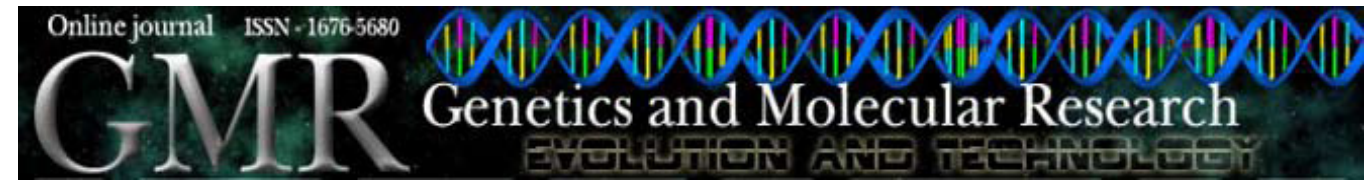

\title{
Molecular and functional analysis of the poly- $\beta$-hydroxybutyrate biosynthesis operon of Pseudomonas sp BJ-1
}

\author{
S.W. Zhu, Z.Y. Fang, H.Y. Jiang and B.J. Cheng \\ School of Life Science, Anhui Agricultural University, Hefei, China \\ Corresponding author: B.J. Cheng \\ E-mail: cbj@ahau.edu.cn
}

Genet. Mol. Res. 9 (4): 2349-2356 (2010)

Received July 9, 2010

Accepted September 3, 2010

Published December 7, 2010

DOI 10.4238/vol9-4gmr845

\begin{abstract}
The operon comprising the genes for poly- $\beta$ hydroxybutyrate (PHB) biosynthesis in Pseudomonas sp BJ-1 was cloned and sequenced. Sequence analysis of 8991 bp revealed that the regions contain two related operons. The first operon contains the three genes $p h b A, p h b B$ and $p h b C$, and the other contains the two genes $f p 1$ and $f p 2$. The deduced amino acid sequences of PHBA and PHBB showed high identity with other bacterial PHB genes. Transcription of the three genes of the first operon is controlled by a single hypothetical promoter region, whereas the other two flp genes are controlled by two hypothetical promoter regions. Analysis of expressed protein at different times showed that PHBA protein levels increased from 0 to $4 \mathrm{~h}$; PHBB and PHBC showed similar kinetics. Detection of enzyme activity showed three proteins with bioactivity and biological function in the synthesis of PHB intermediates.
\end{abstract}

Key words: Poly- $\beta$-hydroxybutyrate; Biosynthesis; Operon; Pseudomonas sp BJ-1 


\section{INTRODUCTION}

Plastic chemicals can hardly be decomposed in nature. They are harmful to human health and the environment (Kaneko et al., 2006). Now, people have become more concerned about protecting the environment. To reduce white pollution, degradable plastic is an inspiring measure to solve the problem, and more scientists are engaged in related research (Agamuthu and Faizura, 2005). Polyhydroxyalkanoates (PHA) are a kind of water-insoluble biodegradable storage polymers (Yan et al., 2006). The most common PHA is poly- $\beta$-hydroxybutyrate (PHB), and this polymer can accumulate up to $90 \%$ of the cellular dry weight of some bacteria (Uchino et al., 2008). PHB as a kind of PHA is a store of polyester in microorganisms and similar to stores of starch or fat in plants or animals. PHB have received increased attention because of their thermoplastic or elastomeric properties, which resemble those of petroleumbased plastics, yet they are completely biodegradable (Chien et al., 2007).

There are many natural bacteria or even plants that can directly produce PHB (Nikel et al., 2006). PHB biosynthesis genes including $p h b A$ (encoding 3-ketothiolase), phbB (encoding acetoacetyl-CoA reductase), $p h b C$ (encoding PHB synthase), phaP, phaR, and phaQ have been cloned from Pseudomonas, Alcaligenes, Streptomyces aureofaciens, Azospirillum brasilense, Rhizobium meliloti, Synechocystis sp, Rhodobacter sphaeroides, Rhodospirillum rubrum (Seo et al., 2003; Lee et al., 2004; Trainer and Charles, 2006; Uchino and Saito, 2006; Wang et al., 2006). Analyses of the PHB operon in many bacteria show that the $p h b A$ and $p h b B$ genes are relatively conserved. Three genes are located in an operon and regulated by identical promoters (Kichise et al., 1999; Matsusaki et al., 2000).

We screened the Pseudomonas sp BJ-1 strain from soil, which possesses high PHB production, and the BJ-1 strain PHB operon was cloned and sequenced. DNA and amino acid sequence alignment confirmed the PHB biosynthesis gene structure and composition. The operon gene array is different from that of other bacteria as well as Pseudomonas sp. PHB was synthesized using acetyl CoA substrates and catalyzed by three protein, which confirms that the PHB operon shows high diversity in different strains.

\section{MATERIAL AND METHODS}

\section{Strain and plasmid}

Pseudomonas sp BJ-1 strain was used in this study. Escherichia coli DH5a strain was grown on $\mathrm{LB}$ medium at $37^{\circ} \mathrm{C}$. The expression plasmid pBV220 was used for gene function validation. E. coli TG1 (Invitrogen) was used as the host for expression plasmid propagation. Ampicillin $(100 \mu \mathrm{g} / \mathrm{mL})$ was added according to the presence of plasmid-borne resistance genes.

\section{PCR amplification and DNA sequencing}

Polymerase chain reaction (PCR) primers were designed based on conserved sites in the PHB operon. The PCR products were cloned into a PGEM-T Easy Vector (Promega). The primers for the PHB operon consisted of forward: CCGGGCCCGCCTTCGCAGGCT and reverse: GGTGTCGCCGAGCAGGATCGA. The vector was sequenced by Applied Biosystems 3730 DNA sequencer at TaKaRa Biotechnology Co., Ltd. Chromatograms were analyzed 
using the Chromas software (Technelysium). Sequence data were deposited in GenBank and the accession No. was AB085816.

\section{ORF prediction and promoter analysis}

To annotate the sequence, putative open-reading frames (ORFs) were identified with ORF finder (http://www.ncbi.nlm.nih.gov/gorf/gorf.html). Initial functional assignments and homology identifications were made by comparison of the translated ORFs to proteins in the BLAST database with BlastP (http://www.ncbi.nlm.nih.gov/BLAST/). Predicted operons, promoters, and terminators were identified with the tools at Softberry (http://www.softberry. com/berry.phtml). ORF nucleotide coding sequence and deduced amino acid sequence were aligned by ClustalW with default parameters.

\section{Protein expression and functional analysis}

The target fragments were ligated to the same restriction sites as of pBV220 vector to obtain $\mathrm{pBV} 220-p h b A$ or $\mathrm{pBV} 220-p h b B$ or $\mathrm{pBV} 220-p h b C$ by standard procedures. The recombinant plasmids were used to transform into $E$. coli TG1 competent cells using the heat shock method. The recombinants were confirmed by restriction enzyme digestion, agarose gel electrophoresis and PCR. E. coli cells were harvested at $4 \mathrm{~h}$ following transfer of the culture plates from $37^{\circ}$ to $42^{\circ} \mathrm{C}$. Cultures maintained at $30^{\circ} \mathrm{C}$ were harvested in parallel as a control. Electrophoresis on a $12.5 \%$ SDS polyacrylamide gel was normally used to analyze the recombinant protein. SDS-PAGE was performed in the Mini-Protean system. After electrophoresis, the gels were stained with Coomassie brilliant blue R250 to visualize the protein bands.

\section{Enzyme activity measurement}

E. coli cells were suspended in $50 \mathrm{mM}$ phosphate buffer, $\mathrm{pH} 7.0$, and then disrupted by ultrasonication at $4^{\circ} \mathrm{C}$. The activities of $\beta$-ketothiolase and acetoacetyl-CoA reductase were assayed according to the method of Oeding and Schlegel (1973). PHB synthase activity was determined according to the modified method of Haywood et al. (1991). Enzyme activity was based on measurements of the product concentrations: CoA and NADPH at $340 \mathrm{~nm}$ and CoA at $412 \mathrm{~nm}$.

\section{RESULTS}

\section{PHB sequence analysis and operon structure}

The entire PHB operon of BJ-1 was sequenced in both strands, and its linkage map is shown in Figure 1. The sequence analysis of the PHB operon (9110 bp) revealed five putative ORFs (Figure 1). Nucleotide sequence analysis revealed that the genes encoding $p h b A$, $p h b B$ and $p h b C$ are separated by $\sim 80 \mathrm{bp}$. This short distance between the genes, which have the same transcription direction, without any promoter region, suggests that $p h b A, p h b B$ and $p h b C$ are co-transcribed. The first ORF ( $p h b C$ ) encoded a 608-amino acid protein, where the entire sequence showed significant sequence identity to known PHB synthesis genes, with $73 \%$ sequence identity with the amidase of the PHA polymerase gene of Burkholderia sp 
DSMZ 9242 strain (Figure 2). The amino acid sequence alignment showed that the $\mathrm{N}$-terminal of the protein is oppositely conserved, where only two Gln are not identical to amidase of the PHA polymerase gene.

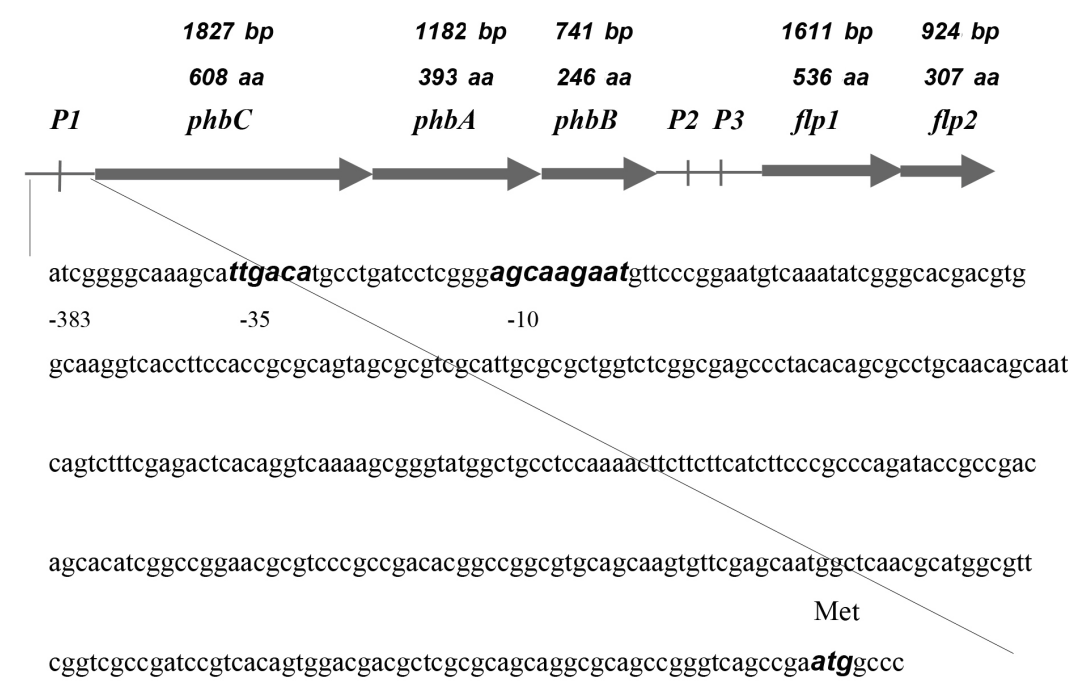

$+1$

Figure 1. Genetic organization of the BJ-1 PHB operon and adjacent region. The number of amino acids and molecular mass are indicated. Sequence from $p h b C$ upstream region is shown. Hypothetical -10 and -35 boxes for $\mathrm{P} 1$ promoter, and the start codon of $p h b C$ are indicated.

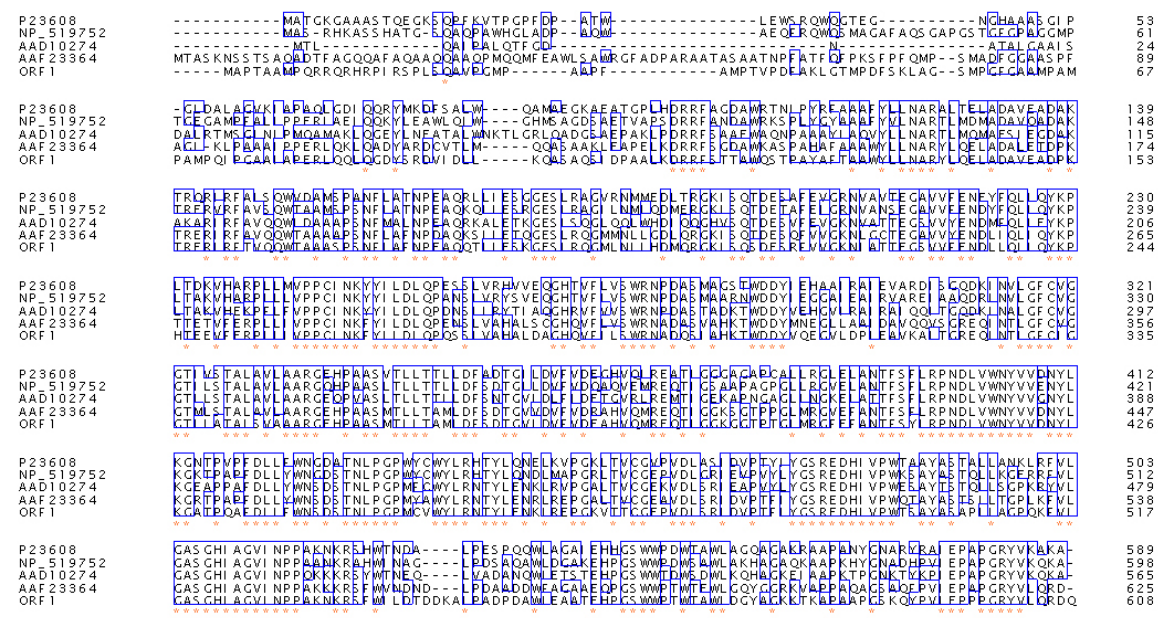

Figure 2. Multiple alignment analysis of open-reading frame 1 (ORF1) and four other protein sequences. AAF23364: phbC in Burkholderia sp DSMZ 9242, 73\% identity to ORF1; P23608: phbC in Ralstonia eutropha, 63\% identity to ORF1; NP_519752: phbC in R. solanacearum, 61\% identity to ORF1; AAD10274: phbC in Alcaligenes latus, $57 \%$ identity to ORF1; ORF1: in this study. 
ORF2 ( $p h b A)$ encoded a 283 -amino acid protein, with $89 \%$ sequence identity with acetyl-CoA acetyltransferase of Burkholderia sp DSMZ 9242 (Figure 3). ORF3 (phbC) encoded a 183-amino acid protein, where BLAST searches indicated that it had significant similarities to acetoacetyl-CoA reductase of many bacteria, showing that ORF3 is the gene of synthesizing enzyme NADPH-dependent acetoacetyl-CoA reductase in Pseudomonas sp BJ-1 (Figure 4). However, ORF4 encoded a 371-amino acid protein, where BLAST searches indicated similarities to MiaB-like tRNA modifying enzyme protein with $91 \%$ sequence identity. The ORF5 gene encoded a 225-amino acid protein, where BLAST searches indicated sequence with high identity to energy metabolism genes in Pseudomonas.

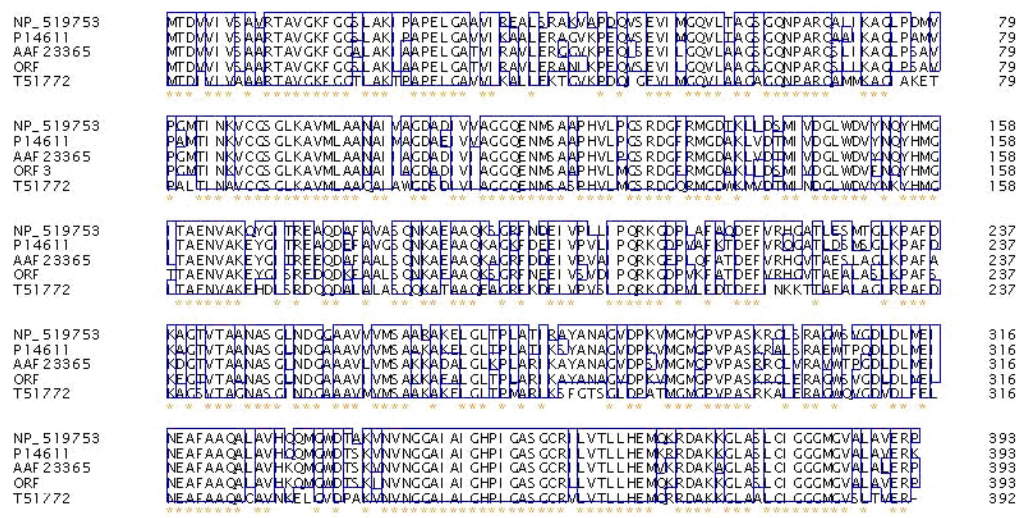

Figure 3. Multiple alignment analysis of open-reading frame 2 (ORF2) and four other protein sequences. NP_519753: phbA in Ralstonia solanacearum, 86\% identity to ORF2; P14611: phbA in R. eutropha, 84\% identity to ORF2; AAF23365: phbA in Burkholderia sp DSMZ 9242, 89\% identity to ORF2; T51772: phbA in Alcaligenes latus, $84 \%$ identity to ORF2; ORF2: in this study.

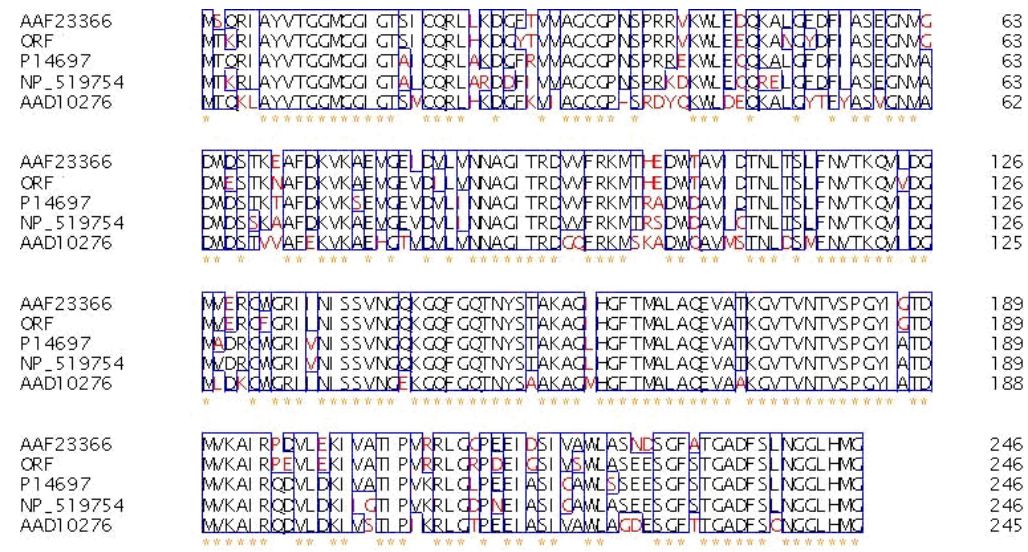

Figure 4. Multiple alignment analysis of open-reading frame 3 (ORF3) and four other protein sequences. AAF23366: Burkholderia sp DSMZ 9242, 91\% identity to ORF3; P14697: Ralstonia eutropha, 85\% identity to ORF3; NP_519754: R. solanacearum, 83\% identity to ORF3; AAD10276: Alcaligenes latus, 75\% identity to ORF3; ORF 3 : in this study. 


\section{Promoter analysis}

The promoter prediction used the promoter database and the Softberry Plant Regulatory motifs database. We named the promotors Pro1, Pro2 and Pro3 (Figure 1). Pro1 controls the expression of ORF1, ORF2 and ORF3, and Pro2 and Pro3 control the expression of ORF4 and ORF5. One of these 5' mRNA termini is located in front of the $p h b C$ gene, and the two promoters are located in front of the $f p 1$ gene. Some other sequences resembling -35 regions TTGACA and -10 regions AGCAAGAAT, are present upstream of the $p h b C$ gene; the biological meaning of these regions needs further studies.

\section{Functional analysis of PHB operon}

To explore whether $p h b$ gene expression would result in the synthesis of PHB, three expression vector constructs were used in the investigation of recombinant protein production in $E$. coli $\mathrm{TG} 1$. The cloned genes were expressed from the tightly regulated temperatureinducible $\mathrm{P}_{\mathrm{R}} \mathrm{P}_{L}$ promoter of pBV220. The results are shown in Figure 5: PHBA, PHBB and PHBC were all expressed at high levels as soluble protein. PHBA showed an enhanced expression of a $42-\mathrm{kDa}$ protein when adapted to growth at a temperature from $30^{\circ}$ to $42^{\circ} \mathrm{C}$. $\mathrm{PHBB}$ showed an enhanced expression of a $26-\mathrm{kDa}$ protein and PHBB expression of a $63-\mathrm{kDa}$ protein when adapted to growth at a temperature from $30^{\circ}$ to $42^{\circ} \mathrm{C}$. The expression of the three proteins confirmed that vector expression was controlled by high temperature.

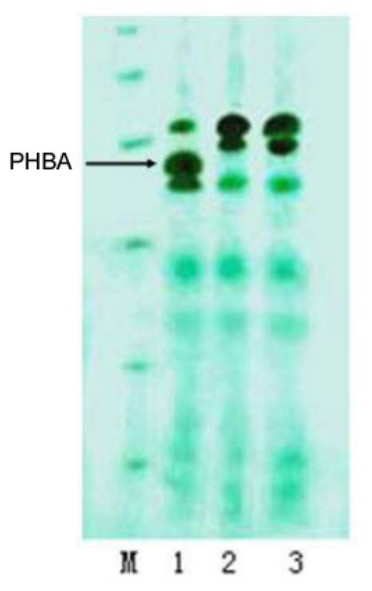

A

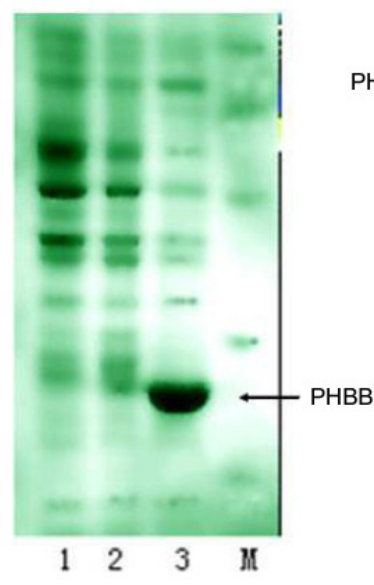

B

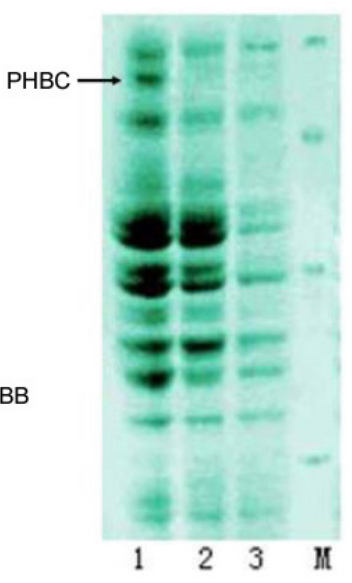

C

Figure 5. SDS-PAGE analysis of expression of $p h b A, p h b B$ and $p h b C$. A. $p h b A$ expression analysis. Lane 1: the expression of $p h b A$ induced by $42^{\circ} \mathrm{C}$; lane 2 : the expression of $p h b A$ induced by $30^{\circ} \mathrm{C}$; lane 3 : the expression of pBV220 induced by $42^{\circ} \mathrm{C}$; M: protein molecular marker. B. phbB expression analysis. Lane 1: the expression of pBV220 induced by $42^{\circ} \mathrm{C}$; lane 2 : the expression of $p h b B$ induced by $30^{\circ} \mathrm{C}$; lane 3 : the expression of $p h b B$ induced by $42^{\circ} \mathrm{C}$; $\mathrm{M}$ : protein molecular marker. C. phbC expression analysis. Lane 1: the expression of $p h b C$ induced by $42^{\circ} \mathrm{C}$; lane 2: the expression of pBV220 induced by $42^{\circ} \mathrm{C}$; lane 3 : the expression of $p h b C$ induced by $30^{\circ} \mathrm{C}$; M: protein molecular marker. 
The effect of the transformed $p h b A, p h b B$ and $p h b C$ genes on PHB biosynthesis in the E. coli recombinants was followed by measuring the intrinsic activity of enzymes involved in the PHB biosynthesis pathway. To investigate the effect of the addition of substrate to PHB enzymes, a series of expression experiments were performed with substrate. As shown in Table 1, the activities of three enzymes $\beta$-ketothiolase, acetoacetyl-CoA reductase and PHB synthase were $0.69,0.58,0.76 \mathrm{units} / \mathrm{mg}$, respectively. The effective enzyme activity showed that the three genes had biological function in the PHB synthesizing approach.

\begin{tabular}{lccc} 
Table 1. Effect of PHB genes on enzyme activities related to PHB biosynthesis. \\
\hline Vector & \multicolumn{3}{l}{ Enzyme activities (units/mg protein) } \\
\cline { 2 - 4 } & $0.69 \pm 0.02$ & $p h b B$ & $p h b C$ \\
\hline pBV220-phbA & - & - & - \\
pBV220-phbB & - & $0.58 \pm 0.01$ & - \\
pBV220-phbC & & - & $0.76 \pm 0.02$ \\
\hline
\end{tabular}

$-=$ not tested.

\section{DISCUSSION}

The production of PHA by different bacteria has drawn attention in recent times because of their use as biodegradable plastic (Liu et al., 2007). There have recently been reports that PHB genes have high sequence difference in different strains (Peralta-Gil et al., 2002). In the present study, we report on the identification and molecular characterization of $p h b A, p h b B$ and $p h b C$, three genes whose nucleic acid sequence contains some singlenucleotide polymorphisms and indels compared to formerly cloned genes. This may be essential for both PHB accumulation and PHA synthase activity in Pseudomonas sp BJ-1.

The three genes are related to PHB biosynthesis by bioinformatics prediction. The operon related to the genes for PHB biosynthesis from Pseudomonas sp BJ-1 was analyzed by protein expression. Many special bacteria have a similar synthesis approach, where five genes are involved in PHB biosynthesis in Alcaligenes eutrophus (Steinbuchel and Schlegel, 1991). We predicted five ORFs by gene prediction software, and protein function was deduced by sequence similarity. ORF1, ORF2 and ORF3 are similar to PHB biosynthesis genes, and ORF1 and ORF2 are conserved with respect to known genes. The three ORF are controlled by the same promoter, confirming that the three genes have identical function. The ORF4 and ORF5 are controlled by Pro4 and Pro5, where the two gene functions are not clear but similar to that of the MiaB-like tRNA modifying enzyme family (Anton et al., 2008). Although the genes are not directly involved with PHB biosynthesis, we presume that the genes are linked to energy metabolism in PHB biosynthesis.

The PHB biosynthesis operon structure and sequence are similar to published operons of other Pseudomonas, but these genes encode a different protein sequence (Takeda et al., 2000). There are two possible reasons for this phenomenon. One is that PHB biosynthesis genes show diversity in different bacteria. Another is that there are several types of PHB biosynthesis operons in bacteria. Different operon genes encode different isozymes, and thus, sequence variation is apparent among these genes. 


\section{ACKNOWLEDGMENTS}

Research supported by the National Transgenic Major Program (\#2009ZX08010002B and \#2008ZX08003-002) and the National 863 Program of China (\#2006AA10Z1B4 and \#2008AA10Z408). We thank Dr. Yong Ding for useful discussions on this study.

\section{REFERENCES}

Agamuthu P and Faizura PN (2005). Biodegradability of degradable plastic waste. Waste Manag. Res. 23: 95-100.

Anton BP, Saleh L, Benner JS, Raleigh EA, et al. (2008). RimO, a MiaB-like enzyme, methylthiolates the universally conserved Asp88 residue of ribosomal protein $\mathrm{S} 12$ in Escherichia coli. Proc. Natl. Acad. Sci. U.S.A. 105: 1826-1831.

Chien CC, Chen CC, Choi MH, Kung SS, et al. (2007). Production of poly-beta-hydroxybutyrate (PHB) by Vibrio spp. isolated from marine environment. J. Biotechnol. 132: 259-263.

Haywood GW, Anderson AJ, Williams DR, Dawes EA, et al. (1991). Accumulation of a poly(hydroxyalkanoate) copolymer containing primarily 3-hydroxyvalerate from simple carbohydrate substrates by Rhodococcus sp. NCIMB 40126. Int. J. Biol. Macromol. 13: 83-88.

Kaneko T, Thi TH, Shi DJ and Akashi M (2006). Environmentally degradable, high-performance thermoplastics from phenolic phytomonomers. Nat. Mater. 5: 966-970.

Kichise T, Fukui T, Yoshida Y and Doi Y (1999). Biosynthesis of polyhydroxyalkanoates (PHA) by recombinant Ralstonia eutropha and effects of PHA synthase activity on in vivo PHA biosynthesis. Int. J. Biol. Macromol. 25: 69-77.

Lee TR, Lin JS, Wang SS and Shaw GC (2004). PhaQ, a new class of poly-beta-hydroxybutyrate (phb)-responsive repressor, regulates phaQ and phaP (phasin) expression in Bacillus megaterium through interaction with PHB. $J$. Bacteriol. 186: 3015-3021.

Liu Q, Ouyang SP, Kim J and Chen GQ (2007). The impact of PHB accumulation on L-glutamate production by recombinant Corynebacterium glutamicum. J. Biotechnol. 132: 273-279.

Matsusaki H, Abe H, Taguchi K, Fukui T, et al. (2000). Biosynthesis of poly(3-hydroxybutyrate-co-3-hydroxyalkanoates) by recombinant bacteria expressing the PHA synthase gene phaC1 from Pseudomonas sp. 61-3. Appl. Microbiol. Biotechnol. 53: 401-409.

Nikel PI, de Almeida A, Melillo EC, Galvagno MA, et al. (2006). New recombinant Escherichia coli strain tailored for the production of poly(3-hydroxybutyrate) from agroindustrial by-products. Appl. Environ. Microbiol. 72: 3949-3954.

Oeding V and Schlegel HG (1973). Beta-ketothiolase from Hydrogenomonas eutropha H16 and its significance in the regulation of poly-beta-hydroxybutyrate metabolism. Biochem. J. 134: 239-248.

Peralta-Gil M, Segura D, Guzman J, Servin-Gonzalez L, et al. (2002). Expression of the Azotobacter vinelandii polybeta-hydroxybutyrate biosynthetic phbBAC operon is driven by two overlapping promoters and is dependent on the transcriptional activator PhbR. J. Bacteriol. 184: 5672-5677.

Seo MC, Shin HD and Lee YH (2003). Functional role of granule-associated genes, phaP and phaR, in poly-betahydroxybutyrate biosynthesis in recombinant $E$. coli harboring phbCAB operon. Biotechnol. Lett. 25: 1243-1249.

Steinbuchel A and Schlegel HG (1991). Physiology and molecular genetics of poly(beta-hydroxy-alkanoic acid) synthesis in Alcaligenes eutrophus. Mol. Microbiol. 5: 535-542.

Takeda M, Kitashima K, Adachi K, Hanaoka Y, et al. (2000). Cloning and expression of the gene encoding thermostable poly(3-hydroxybutyrate) depolymerase. J. Biosci. Bioeng. 90: 416-421.

Trainer MA and Charles TC (2006). The role of PHB metabolism in the symbiosis of rhizobia with legumes. Appl. Microbiol. Biotechnol. 71: 377-386.

Uchino K and Saito T (2006). Thiolysis of poly(3-hydroxybutyrate) with polyhydroxyalkanoate synthase from Ralstonia eutropha. J. Biochem. 139: 615-621.

Uchino K, Saito T and Jendrossek D (2008). Poly(3-hydroxybutyrate) (PHB) depolymerase PhaZa1 is involved in mobilization of accumulated PHB in Ralstonia eutropha H16. Appl. Environ. Microbiol. 74: 1058-1063.

Wang C, Meek DJ, Panchal P, Boruvka N, et al. (2006). Isolation of poly-3-hydroxybutyrate metabolism genes from complex microbial communities by phenotypic complementation of bacterial mutants. Appl. Environ. Microbiol. 72: 384-391.

Yan S, Tyagi RD and Surampalli RY (2006). Polyhydroxyalkanoates (PHA) production using wastewater as carbon source and activated sludge as microorganisms. Water Sci. Technol. 53: 175-180. 